NOTICE: this is the author's version of a work that was accepted for publication in Mathematics and Computers in Simulation. Changes resulting from the publishing process, such as peer review, editing, corrections, structural formatting, and other quality control mechanisms may not be reflected in this document. Changes may have been made to this work since it was submitted for publication. A definitive version was subsequently published in Mathematics and Computers in Simulation, Vol. 93 (2013). DOI: 10.1016/j.matcom.2013.04.010 


\title{
Measuring bias in a term-structure model of commodity prices through the comparison of simultaneous and sequential estimation
}

\author{
Hiroaki Suenaga ${ }^{a}$ \\ a School of Economics and Finance, Curtin University, GPO Box U1987, Perth, WA 6845, \\ Australia. email: hiroaki.suenaga@cbs.curtin.edu.au, phone: +61-8-9266-4480, fax: +61-8-9266- \\ 3026.
}

\begin{abstract}
This study examines bias in a term-structure model of commodity prices in specifying the true stochastic dynamics of underlying spot price. The bias is quantified by comparing the model estimated by the conventional method of estimating all model parameters simultaneously with a panel of futures prices and the model estimated by an alternative method of estimating model parameters in two steps. In this alternative approach, a subset of model parameters is first estimated on the first difference of observed futures prices so that these parameters are free from bias in specifying deterministic price variation and the dynamics of the underlying state variables. In the second step, the remaining model parameters are estimated on the futures price equations, while holding the parameters estimated in the first step. Empirical applications to four commodities (gold, crude oil, natural gas, and corn) reveal that the two-factor model widely considered in the literature is subject to a misspecification bias of substantial size. Out-of-sample forecast test indicates that, for three of the four commodities considered, the model estimated by the sequential method yields a considerably more accurate price forecast than the model estimated by the simultaneous method.
\end{abstract}

Keywords: commodity prices, term-structure model, volatility, 


\section{Introduction}

Recent increase in the level and volatility of oil, metals, and other primary commodity prices has raised the importance of a better understanding of the stochastic properties of these commodity prices and tools to hedge against price risks. Stochastic dynamics of commodity prices and pricing of derivative contracts have been studied extensively in the field of financial economics. The standard approach in this literature is to specify the stochastic dynamics of an underlying asset, usually the spot price of the commodity under investigation, by a series of stochastic differential equations, and derive from the suggested model valuation formulas of various derivative contracts whose payoff depends on the value of the underlying asset realized at the contract maturity date [7].

Recent advancements in this modeling approach have been attained through increasing the number of state variables to specify the stochastic dynamics of the underlying spot price and/or stipulating more complex stochastic process of each state variable. These flexible models generally exhibit better fit to the observed price data. Yet, it is often understated that these models only approximate true stochastic dynamics of commodity prices. In particular, the theory of storage illustrates that, for a commodity with significant storage cost and seasonality in demand and/or supply, an equilibrium path of spot and futures prices is highly non-linear and cannot be expressed in a reduced form even in a simulation setting [25]. Thus, no matter how flexible they are specified, models specifying the dynamics of commodity prices are intrinsically subject to approximation bias.

The main objective of this study is twofold. First, this study examines bias in a two-factor term-structure model of commodity prices widely considered in the literature. The bias is measured by comparing the model estimated by two different methods. The first method, the one standard in the literature, is to estimate all model parameters simultaneously with a panel of observed futures prices. The second method estimates a subset of model parameters that appear on the first difference of observed futures prices. First differencing eliminates the dynamics of the underlying state variables and leaves only the innovation errors in the estimation equation. It also eliminates seasonal and other deterministic price variation from

\footnotetext{
${ }^{1}$ A literature on the dynamics of oil and petroleum commodity prices has been growing. However, many studies commonly examine, either separately or jointly with the spot price series, a single time series constructed by splicing nearby futures prices (see, for example, Lee et al. [9] and Chang et al. [3]). In contrast, term-structure models link the spot price to multiple futures prices observed per day through no arbitrage. The focus of this paper is centered on the evaluation of these term-structure models in their implications for price variances and cross-sectional correlation.
} 
the estimation equation. Thus, it allows estimation of the key model parameters without imposing a priori restrictions on the dynamics of the state variables and deterministic price variation. The bias in specifying these dynamics is captured by the idiosyncratic error included in the right-hand side of the futures price equation in the standard approach of estimating all model parameters simultaneously with a panel of futures prices. Thus, the magnitude of this misspecification bias is measured through comparing the estimated variance of the idiosyncratic error between the two estimation methods.

The second objective of this study is to propose a sequential procedure to estimate parameters in a term-structure model of commodity prices. In this alternative approach, a subset of model parameters is first estimated on the first difference of observed futures prices so that the estimated parameters are free from bias in specifying deterministic price variation and the dynamics of the state variables. In the second step, the remaining parameters, those determining seasonal mean prices and deterministic variation in the state variables, are estimated on the futures price equations while holding the parameters estimated in the first step. The proposed sequential method is evaluated empirically by its ability to forecast subsequent period futures prices, relative to the forecast based on the model estimated by the standard method of estimating all model parameters simultaneously.

Empirical applications of the model to four commodities with different characteristics (crude oil, natural gas, gold, and corn) illustrate that the conventional approach of estimating all model parameters simultaneously yields the variance of the idiosyncratic error that is substantially greater than the variance estimated on the first difference model. The result indicates that deterministic price variation and the dynamics of the state variables as specified in a conventional two-factor model are subject to an approximation bias of substantial size. The estimates of other model parameters also differ considerably between the two estimation methods. In particular, the simultaneous estimation method yields substantially lower seasonal mean price estimates and predicts higher values of the state variables for natural gas and gold than the sequential estimation method. Out-of-sample forecast test shows that the model estimated by the sequential method yields considerably more accurate forecast than the model estimated by the simultaneous method for natural gas, gold, and corn. For crude oil, the two estimation methods yield the forecasts with roughly the same accuracy.

The next section reviews the conventional approach to modeling the term structure of commodity prices and illustrates that this modeling approach is inherently subject to approximation bias. The section then proposes an alternative approach to the estimation of 
the model parameters. Section three estimates a two-factor term structure model with data from four commodity markets and examines the model's bias in approximating the true price dynamics by comparing the estimated model parameters between the two estimation methods. The section also compares the two estimation methods by their relative accuracy in forecasting out-of-sample data. Section four concludes the paper.

\section{Bias in Term-Structure Models of Commodity Prices}

A conventional approach to modeling term structure of commodity prices can be exemplified by the two-factor model of Sorensen [19]. In this model, the log spot price of the commodity is specified as a linear combination of three components; seasonal mean price and long-term (LT) and short-term (ST) deviation from seasonal mean price. The model takes the following form, ${ }^{2}$

$$
\begin{aligned}
& \ln S_{t}=f(t ; \mathbf{b})+x_{1, t}+x_{2, t} \\
& d x_{1, t}=\mu d t+\sigma_{1} d w_{1} \\
& d x_{2, t}=-\kappa x_{2, t} d t+\sigma_{2} d w_{2} \\
& d w_{1} d w_{2}=\rho d t
\end{aligned}
$$

where $S_{t}$ is the spot price in period $t ; f(t ; \mathbf{b})$ represents seasonal or other deterministic variation in the spot price; $x_{1, t}$ and $x_{2, t}$ are the state variables representing, respectively, the LT and ST deviation from the seasonal mean price; $d w_{1}$ and $d w_{2}$ are increments to standard Brownian motions; and $\mathbf{b}, \mu, \kappa, \sigma_{1}$, and $\sigma_{2}$ are the parameters determining, respectively, the seasonal mean price, drift rate, mean reversion rate, and diffusion rate of the two stochastic factors.

The price in period $t$ of the futures contract that matures in period $T$ is obtained as the period $t$ conditional expectation, under the risk-neutral probability measure, of the spot price in $T$. For the spot price following the stochastic process in (1), the pricing formula of this futures contract is obtained as, ${ }^{3}$

$$
\ln F_{T, t}=f(T ; \mathbf{b})+A(\tau)+x_{1, t}+x_{2, t} e^{-\kappa \tau}
$$

\footnotetext{
${ }^{2}$ Sorensen [19] applies the model (1) to the term structure of three agricultural commodity futures (corn, wheat, and soybean). Manoliu and Tompaidis [12] and Lucia and Schwartz [11] consider the same model in their analyses of natural gas and electricity prices, respectively.

${ }^{3}$ The pricing formula (2) assumes constant market price of risks. See Sorensen [19] for more details.
} 
where $A(\tau)=\left(\mu-\lambda_{1}+\frac{\sigma_{1}^{2}}{2}\right) \tau+\frac{\sigma_{2}^{2}\left(1-e^{-2 \kappa \tau}\right)}{4 \kappa}-\frac{\left(\lambda_{2}-\rho \sigma_{1} \sigma_{2}\right)\left(1-e^{-\kappa \tau}\right)}{\kappa}, \tau=T-t$ is the time-tomaturity, and the two coefficients $\lambda_{1}$ and $\lambda_{2}$ are generally referred as the market price of risk associated with the corresponding state variable.

The set of parameters defining model (1), $\mathbf{\Omega}=\left\{\mathbf{b}, \kappa, \sigma_{1}, \sigma_{2}, \lambda_{1}, \lambda_{2}, \rho\right\}$, is usually estimated with futures price data. To fit equation (2) into multiple prices with different maturity dates observed per day, an error term, often called the measurement error, is added to the right-hand side of (2), which makes the values of the two state variables $x_{1}$ and $x_{2}$ not identifiable. The model is thus characterized econometrically as a latent factor model, which is commonly estimated through a filtering method. The model is represented in the following state-space form

$$
\begin{aligned}
& \ln F_{T, t}=f(T ; \mathbf{b})+A(\tau)+x_{1, t}+e^{-\kappa \tau} x_{2, t}+u_{T, t} \\
& x_{1, t}=\mu+x_{1, t-1}+v_{1, t} \\
& x_{2, t}=e^{-\kappa} x_{2, t-1}+v_{2, t}
\end{aligned}
$$

... Measurement equation

... Transition equation

where $u_{T, t}$ is the measurement error with $\mathrm{E}\left[u_{T, t}\right]=0, \mathrm{~V}\left[u_{T, t}\right]=\sigma_{T, t}^{2}$, and $\mathrm{E}\left[u_{T, t}, u_{S, s}\right]=0$ for all $t \neq S$ and/or $T \neq S$. In (4), the vector of innovation errors $\mathbf{v}_{t}=\left\{v_{1, t}, v_{2, t}\right\}^{\prime}$ is serially uncorrelated and identically distributed with $\mathbf{v}_{t} \sim \mathrm{N}(0, \mathbf{H})$ for all $t$ where $\mathbf{H}$ is a 2 by 2 symmetric matrix with $\sigma_{1}^{2}$ and $\sigma_{2}^{2}$ on the main diagonal and $\rho \sigma_{1} \sigma_{2}$ off diagonal.

Term-structure models similar to (1) have been considered widely in the literature. ${ }^{4}$ For example, Gibson and Schwartz [6] and Nielsen and Schwartz [14] consider a model similar to (1) in analyzing oil and copper, yet without a seasonal variation in mean price. ${ }^{5}$ Models with more than two factors are also suggested in the literature. For example, Schwartz [16] extends the model (1) by adding a third factor representing the stochastic interest rate and applies it to

\footnotetext{
${ }^{4}$ See Lautier [8] for a comprehensive review on applications of term-structure models to various commodities.

${ }^{5}$ These two studies also parameterize the stochastic process of two factors differently from (1) so that the two factors are interpreted as representing the spot price and convenience yield factor. Schwartz and Smith [17] illustrate that, aside from the absence of seasonal variation in mean price, these models are equivalent to model (1).
} 
gold, copper, and crude oil. ${ }^{6}$ Casassus and Collin-Dufresne [2] also consider a three-factor model, yet allow the three factors to follow a more flexible stochastic process than Schwartz [16]. Cortazar and Naranjo [4] extend the model into a general $n$-factor setting, with each factor assumed to follow a Gaussian process.

With an increased number of state variables and/or more complex stochastic process stipulated on each variable, these flexible models generally exhibit better fit to the observed data. However, it is often understated that these models, even recently developed flexible models, only approximate the true stochastic dynamics of commodity prices. As shown by the theory of storage $[15,25]$, for a commodity with significant storage cost and seasonality in demand or supply, an equilibrium path of spot and futures prices exhibits highly non-linear dynamics. In particular, prices of concurrently traded futures contracts with different maturity dates are linked through inter-temporal arbitrage, yet this inter-temporal price link breaks at the end of demand year when discretionary inventory is driven to zero. This implies that cross-sectional price correlation across concurrently traded contracts varies discontinuously across season. ${ }^{7}$ Stochastic processes of the underlying spot price stipulated in models of commodity price dynamics, even recently developed flexible models, cannot induce a futures price formula that replicates these complex dynamics of commodity futures prices.

Bias in approximating the true price dynamics can be very large for models imposing strong restrictions on the dynamics of the underlying spot price. The magnitude of this bias cannot be quantified either empirically or theoretically in the absence of a reduced-form expression of an equilibrium price path. One way to measure this bias, as proposed in this paper, is to compare the model defined in (3) and (4) with its first difference form,

$$
\Delta \ln F_{T, t}=\ln F_{T, t}-\ln F_{T, t-1}=B(\tau)+v_{1, t}+e^{-\kappa \tau} v_{2, t}+\Delta u_{T, t}
$$

\footnotetext{
${ }^{6}$ Other studies applying three factor models include Miltersen and Schwartz [13] for copper, Bhar and Lee [1] and Cortazar and Schwartz [5] for crude oil, Liu and Tang [10] and Tang [23] for crude oil and copper, and Todorova [24] for oil and natural gas. Cortazar and Schwartz [5] and Todorova [24] employ different parameterization from Schwartz [16] and interpret three factors as representing LT, ST, and convenience yield factor.

${ }^{7}$ This highly non-linear price dynamics and other features such as time-to-maturity effects and strong seasonality in price volatility are depicted by Smith [18] for corn, Suenaga et al. [22] for natural gas, and Suenaga and Smith [21] for crude oil and refined petroleum commodities.
} 
where $B(\tau)=\lambda_{1}-\frac{\sigma_{1}^{2}}{2}-\frac{\sigma_{2}^{2} e^{-2 \kappa \tau}\left(1-e^{-2 \kappa}\right)}{4 \kappa}+\frac{\left(\lambda_{2}-\rho \sigma_{1} \sigma_{2}\right) e^{-\kappa \tau}\left(1-e^{-\kappa}\right)}{\kappa} . \operatorname{In}(5), \Delta u_{T, t}=u_{T, t}-u_{T, t-1}$ with $\mathrm{E}\left[\Delta u_{T, t}\right]=0, \mathrm{~V}\left[\Delta u_{T, t}\right]=\sigma_{T, t}^{2}+\sigma_{T, t-1}^{2}$, and $\mathrm{E}\left[\Delta u_{T, t}, \Delta u_{S, S}\right]=0$ for all $t \neq s$ and/or $T \neq S$ given the distributional assumption of $u_{T, t}$.

With the assumption that the innovations to the two state variables follow standard Brownian motions, the vector of innovation errors in (5) is serially uncorrelated and identically distributed with $\mathbf{v}_{t} \sim \mathrm{N}(0, \mathbf{H})$ where $\mathbf{H}$ is as defined before. Thus, the vector of coefficients appearing on the model (5), $\Omega_{1}=\left\{\sigma_{1}, \sigma_{2}, \lambda_{1}, \lambda_{2}, \rho\right\}$, can be estimated by the method of maximum likelihood.

As is clear from the comparison of the first difference form (5) with the original futures price equations (3) and (4), first differencing eliminates the term $f(t ; \mathbf{b})$ from (5). The absence of the drift parameter $\mu$ in (5) also indicates that the first differencing eliminates any deterministic variations in the dynamics of the two state variables $x_{1}$ and $x_{2}$. The two latent factors $v_{1}$ and $v_{2}$ in (5) are the innovations to the state variables. Thus, the first difference form (5) allows the possibility that the dynamics of the two state variables involve deterministic variations more flexible than those assumed in (1). The stochastic dynamics of the two state variables are also not explicit in (5). They remain only partly and implicitly in (5) by restricting the factor loadings that link two latent factors $v_{1}$ and $v_{2}$ to observed futures prices. Specifically, the loadings of the LT factor are unity for all contracts and for all trading days whereas those of the ST factor decay exponentially with time-to-maturity $\tau$ at the rate of $\exp (-\kappa)$ due to the specification that $x_{1}$ and $x_{2}$ follow a BM with drift and the MR process, respectively. Because the dynamics of $x_{2}$ is not explicit in the first difference form (5), the value of $\kappa$ is determined by the cross-sectional differences in the price movements of concurrently traded contracts in estimation of (5), rather than being estimated on the stipulated stochastic dynamics of $x_{2}$.

Since the first difference form (5) avoids specifying seasonal and other deterministic price variation and the dynamics of the two state variables, the parameter vector $\boldsymbol{\Omega}_{1}$ estimated on (5) is free from bias in specifying these dynamics. These two sources of bias can be quantified by comparing the estimate of $\boldsymbol{\Omega}_{1}$ with the corresponding parameters estimated on the original futures price equations (3) and (4). In particular, the two sources of bias interact with each other and are reflected in the measurement error on the right-hand side of (3). Thus, 
comparing the variance of the measurement error estimated on (5) with the one estimated on (3) and (4) provides a measure of the two sources of bias.

The first difference form (5) also facilitates an alternative approach to the estimation of term-structure models of commodity prices. In this approach, the model parameters are estimated in two steps. In the first step, a subset of the parameters that appear in the first difference form is estimated. In the second step, the remaining parameters are estimated on the futures price equations, while holding the other parameters at their estimates from the first step. For the two-factor model defined in (1), as an example, the set of parameters $\boldsymbol{\Omega}_{1}=\left\{\sigma_{1}, \sigma_{2}, \lambda_{1}, \lambda_{2}, \rho\right\}$ is first estimated on the first difference form (5). In the second step, the remaining parameters $\Omega_{2}=\{\mathbf{b}, \boldsymbol{\kappa}\}$ are estimated on the futures price equations (3) and (4), holding $\boldsymbol{\Omega}_{1}$ at its estimate from the first step. The estimate of $\boldsymbol{\Omega}_{1}$ from the first step of this sequential procedure is free from bias in specifying $f(t ; \mathbf{b})$ and the deterministic variations of the two state variables. In the second step, equations (3) and (4) are subject to bias in specifying $f(t ; \mathbf{b})$ and the dynamics of $x_{1}$ and $x_{2}$. However, by holding $\boldsymbol{\Omega}_{1}$ at its estimate from the first step, $\mathbf{\Omega}_{2}=\{\mathbf{b}, \boldsymbol{\kappa}\}$ is estimated without being affected by the bias in the estimate of $\boldsymbol{\Omega}_{1}$ in the second step of the sequential procedure.

\section{Empirical Applications}

In this section, the two-factor term-structure model defined in (1) is estimated with empirical data. The model is estimated in its original state-space form (3) and (4) as well as in its first difference form (5), and the magnitude of the model's misspecification bias is examined by comparing the model estimated in the two forms. The section also compares the suggested sequential estimation method with the conventional estimation method by their ability to forecast futures prices in subsequent periods.

\subsection{Data}

The two-factor term-structure model of commodity prices defined in (1) is estimated in its original state-space form (3) and (4) and its first difference form (5). The model is estimated with the empirical data from the markets for the following four commodities with different characteristics:

- Natural gas - consumption commodity with strong seasonality in demand,

- Corn - consumption commodity with strong seasonality in supply, 
- Crude oil - consumption commodity with very weak seasonality in demand and supply, and

- Gold - investment commodity with virtually no seasonality either in demand or supply.

The model is estimated with the daily settlement prices of futures contracts traded at the New York Mercantile Exchange (crude oil, natural gas, and gold) and Chicago Board of Trade (corn). The sample period starts on January 1, 1984, for corn and gold; on April 1, 1984, for crude oil; and on April 1, 1991, for natural gas and ends on September 1, 2009, for all four commodities. For each of the four commodities, the model is estimated with the data up to December 31, 2007. ${ }^{8}$ The last twenty months of data are used for an out-of-sample forecast test. Because long-dated contracts do not trade actively, the prices of contracts of more than twelve months to maturity are excluded both from the model estimation and from the forecasting test, except that prices of contracts of no more than eighteen months to maturity are analyzed for corn. Excluding these observations leaves 75,882 prices among 329 contracts for crude oil, 57,960 prices among 245 contracts for natural gas, 46,628 prices among 179 contracts for gold, and 52,131 prices among 151 contracts for corn. ${ }^{9}$

\subsection{Model specification}

In estimating term-structure models similar to (1), previous studies commonly specify that the variance of the measurement error varies by the delivery month of the contract and not by the time-to-maturity or by the trade date. The specification simplifies the variance of the measurement error on the right-hand side of (3) to $\sigma_{T, t}^{2}=\sigma_{m(T)}^{2}$ where $m(T)$ converts the maturity date $T$ into the contract delivery month. Suenaga [20] illustrates that this simple variance structure is empirically not supported and a more flexible variance structure is necessary to replicate the complex volatility dynamics for all four commodities examined in this study. Given this finding, I specify the variance of the measurement error by a flexible function as in (6) so that the variance can vary both by time-to-maturity and by the delivery month of the contract, 10

\footnotetext{
${ }^{8}$ The model is estimated with the data prior to the global financial crisis (GFC). The two-factor Gaussian model defined in (1) is not specified to accommodate large price swings experienced during the GFC. Thus, estimating the model with the data inclusive of the GFC period would imply even greater approximation bias than the level reported in Section 3.3.

${ }^{9}$ Of these observations, 70,800 prices (307 contracts), 52,780 prices (223 contracts), 43,820 prices (168 contracts), and 48,762 prices (142 contracts) are used to estimate the model for crude oil, natural gas, gold, and corn, respectively.

${ }^{10}$ Although the results are not reported here, I also estimated the model with the simple variance structure $\sigma_{T, t}^{2}=\sigma_{m(T)}^{2}$ commonly employed in the literature. Both Akaike Information Criterion and
} 


$$
\sigma_{T, t}^{2}=\exp \left[\gamma_{m(T), 0}+\gamma_{m(T), 1} d+\sum_{k=1}^{3}\left(\gamma_{m(T), 2 k} \sin \left(\frac{2 \pi k d}{d_{\max }}\right)+\gamma_{m(T), 2 k+1} \cos \left(\frac{2 \pi k d}{d_{\max }}\right)\right)\right]
$$

where $d=T-t$ is the time-to-maturity of the contract and $d_{\max }$ is the maximum days to maturity for which the model is estimated (one year or $d_{\max }=252$ trading days for crude oil, natural gas, and gold, and eighteen months or $d_{\max }=378$ trading days for corn).

A common specification in the literature for the deterministic price variation $f(t ; \mathbf{b})$ is to allow the seasonal mean price to vary by the delivery month of the contract. The specification is implemented with a set of dummy variables as follows,

(7) $\quad f(s ; \mathbf{b})=\sum_{k \in K} b_{k} z_{k, s}$

where $z_{k, s}$ is the dummy variable which takes the value one if the maturity date $s$ of the futures contract corresponds to the delivery month $k$ and takes the value zero otherwise. I employ the same specification as (7) in estimating the model (3) and (4). Of the four commodities examined in this paper, crude oil and natural gas have a contract maturing each month, gold has six contracts maturing each year (Feb, Apr, June, Aug, Oct, and Dec), and corn has five contracts maturing each year (Mar, May, July, Sep, and Dec). Thus, I set $K=\{1, \ldots, 12\}$ for crude oil and natural gas, $K=\{2,4,6,8,10,12\}$ for gold, and $K=\{3,5,7,9,12\}$ for corn.

\subsection{Estimation results}

It is illustrated in Section 2 that the errors in specifying deterministic price variation and the dynamics of the two state variables are quantified by comparing the estimates of the variance of the measurement error between the two estimation methods. Figures 1 through 4 plot the variance of the measurement error calculated based on the estimate of the variance function (6) obtained by the two estimation methods. In each figure, the variance is computed for each contract delivery month $m(T)$ over a one-year trading horizon (18 months for corn) and is aligned with the trading date. In panel (a) of Figures 1 through 4 , the variance

Schwartz Information Criterion support the model allowing flexible variance structure of (6) over the restrictive specification. The specification of variance structure of the measurement error, however, does not alter the main results reported in the rest of this paper. 
estimated on the first difference form (5) exhibits a strong time-to-maturity effect. That is, for all four commodities, the variance of the measurement error increases rapidly as the contract approaches its maturity date. The variance also indicates strong seasonality for the three consumption commodities. For natural gas, the variance in the last two months of trading is highest for contracts maturing in winter when demand peaks for space heating. For crude oil, the variance is high for contracts maturing in summer and winter. For corn, the variance is particularly high for the July contract, because the low inventory right before the arrival of a new harvest does not allow unexpected price shocks to be absorbed through the inventory adjustment. The variance of the measurement error is also high for the October contract of gold, which is known to have a substantially low trading volume relative to the other five contracts.

\section{[FIGURES 1-4 SOMEWHERE HERE]}

In panel (b) of Figures 1 through 4, the variance of the measurement error estimated on the futures price equations (3) and (4) replicates a similar volatility pattern (seasonality and time-to-maturity effect), yet it is substantially greater than the variance estimated on the first difference form. For crude oil, the variance of the measurement error estimated on the futures price equations, on average over one year of trading and over twelve contracts, is about 17.6 times greater than the variance estimated on the first difference form. For the other commodities, the variance estimated on the futures price equations is on average 91.4, 21.2, and 102.4 times greater than the estimate on the first difference form for natural gas, gold, and corn, respectively. These results indicate that the errors in specifying deterministic price variation and the dynamics of the two state variables are of substantial magnitude in the twofactor model (1) applied to the four commodities. The size of this bias is surprising for all four commodities and particularly for gold, for which price exhibits no systematic variation and concurrently traded contracts exhibit high price correlation.

Tables 1 through 4 report the estimates of the other parameters in model (1). In each table, panels (a) and (b) report the estimates obtained by the simultaneous method and those obtained by the sequential method, respectively. There are several interesting observations in comparing the results obtained by the two estimation methods. First, the coefficient estimates for the seasonal mean prices can differ substantially between the two estimation methods. In particular, the sequential method yields substantially greater mean price estimates for natural gas and gold and lower estimates for corn than the simultaneous estimation. For crude oil, 
the estimated mean prices are larger but only slightly so for the sequential method than for the simultaneous method.

\section{[TABLES 1-4 SOMEWHERE HERE]}

These differences in the estimates of the seasonal mean prices imply that the sequential estimation predicts lower average values of the state variables for natural gas and gold and higher average values for corn than the simultaneous method. This is indeed the case and verified in Table 5, which reports the sample mean and standard deviation of the state variables predicted through the Kalman filter. For natural gas, the sample mean of the predicted LT factor is substantially greater for the simultaneous estimation than for the sequential estimation ( 0.623 and 0.471 , respectively). For gold, the predicted LT factor is smaller for the simultaneous estimation (-1.331) than for the sequential estimation $(-0.755)$. However, the sample mean of the predicted ST factor is greater for the former $(0.879)$ than for the latter $(-0.042)$ by a magnitude greater than the difference in the predicted LT factor. For corn, the predicted ST factor is substantially smaller for the simultaneous estimation $(-0.138)$ than for the sequential estimation (-0.019). The estimates of the drift parameter $\mu$ are also consistent with the predicted values of the LT factor. The simultaneous estimation method yields a substantially higher (lower) estimate of the drift rate than the sequential method for natural gas and corn (gold), for which the predicted values of the LT factor are substantially higher (lower) by the former than the latter estimation method.

\section{[TABLE 5 SOMEWHERE HERE]}

The second interesting observation in Tables 1 through 4 is that, for all four commodities, the estimated mean-reversion coefficient $\kappa$ is very small, implying a very high persistence of the ST factor. The estimated mean-reversion rates translate into an autoregressive coefficient of close to one and slightly higher for the simultaneous estimation than for the sequential estimation. A well-known downward bias in the estimate of the autoregressive coefficient predicts that the simultaneous estimation of the mean-reversion rate is biased upward yet the bias is almost negligible due to a large sample size. The results shown in the tables oppose this prediction. For all four commodities, the estimated mean reversion rate is higher (implying lower persistence) for the sequential estimation than for the simultaneous estimation. This result indicates that the cross-sectional differentials in the observed daily price changes imply a lower persistence of price shocks than the stochastic process of the underlying spot price as estimated with a panel of the observed futures prices. 
Third, the estimates of the two diffusion parameters $\sigma_{1}$ and $\sigma_{2}$ are of similar magnitudes for the two estimation methods, except that, for gold, $\sigma_{2}$ estimated on the futures price equations (3) and (4) is much greater than the same coefficient estimated on the first difference form (5). This result is consistent with the distribution of the predicted factors. In Table 5, the sample standard deviations of the predicted factors differ only slightly between the two estimation methods for crude oil, natural gas, and corn. For gold, however, the sample standard deviation of the predicted ST factor is substantially greater for the simultaneous estimation than for the sequential estimation.

From equation (5), the variance of the daily futures returns is obtained as:

$$
\mathrm{V}[\Delta \ln F(t, T)]=\sigma_{1}^{2}+e^{-2 \kappa \tau} \sigma_{2}^{2}+2 e^{-\kappa \tau} \rho \sigma_{1} \sigma_{2}+\sigma_{T, t}^{2}
$$

The sum of the first three terms on the right-hand side of (8) represents the variance of the price shocks attributable to the two common factors, which depends on the two diffusion parameters $\left(\sigma_{1}\right.$ and $\left.\sigma_{2}\right)$, the mean reversion parameter $(\kappa)$, and time-to-maturity $(\tau)$. This component is computed for each of the four commodities, based on the three parameters estimated by each of the two estimation methods. Figure 5 shows how it changes over a oneyear trading horizon (eighteen months for corn). In the figure, the variance attributable to the two common factors decreases exponentially with the time-to-maturity for all four commodities, due to the MR process stipulated on the ST factor. For crude oil, natural gas, and corn, these dynamics are almost identical for the two estimation methods, except that the rate of this decrease is slightly higher for the sequential estimation than for the simultaneous estimation due to the lower mean-reversion coefficient estimated by the former method than by the latter. For gold, the variance attributable to the two common factors is substantially higher for the simultaneous estimation than for the sequential estimation because the estimated diffusion parameter of the ST factor is substantially higher for the former than for the latter method. This difference in the variance of the ST factor implied by the two estimation methods represents the bias in specifying the deterministic price variation and the dynamics of the two state variables. For the other three commodities, these biases are almost all captured by the measurement error. For gold, a dominant share of the bias is captured by the measurement error, yet a considerable amount of the bias is also captured by the ST factor, most likely because the bias is correlated across concurrently traded contracts.

[FIGURE 5 SOMEWHERE HERE] 
Finally, in Tables 1 through 4 , the estimates of the market price of risk parameters are very small for all four commodities and for both estimation methods. Interestingly, some of these coefficients are found significant by the simultaneous estimation, due primarily to the small standard errors. In contrast, the estimated market price of risk parameters are insignificant for all commodities by the sequential estimation method.

\subsection{Forecasting test}

In this section, I compare the model estimated by the two methods by their relative accuracy in forecasting out-of-sample futures prices. The price in period $t_{1}$ of the futures contract maturing at $T_{1}$ is given by (3) as,

$$
\ln F_{T_{1}, t_{1}}=f\left(T_{1} ; \mathbf{b}\right)+A\left(\tau_{1}\right)+x_{1, t_{1}}+e^{-\kappa \tau_{1}} x_{2, t_{1}}+u_{T_{1}, t_{1}}
$$

where $\tau_{1}=T_{1}-t_{1}$. A forecast in period $t_{0}\left(t_{0}<t_{1}\right)$ of this futures price is constructed as the expected value of $\ln F_{T_{1}, t_{1}}$ conditional on the information available at $t_{0}$, i.e., $E\left[\ln F_{T_{1}, t_{1}} \mid \mathfrak{J}^{t_{0}}\right]$ where $\mathfrak{J}^{t_{0}}$ denotes the set of information available at $t_{0}$. This conditional expectation is obtained as,

$$
\begin{aligned}
\mathrm{E}\left[\ln F_{T_{1}, t_{1}} \mid \mathfrak{J}^{t_{0}}\right] & =f\left(T_{1} ; \mathbf{b}\right)+A\left(\tau_{1}\right)+\mathrm{E}\left[x_{1, t_{1}} \mid \mathfrak{J}^{t_{0}}\right]+e^{-\kappa \tau_{1}} \mathrm{E}\left[x_{2, t_{1}} \mid \mathfrak{I}^{t_{0}}\right] \\
& =f\left(T_{1} ; \mathbf{b}\right)+A\left(\tau_{1}\right)+\mu\left(t_{1}-t_{0}\right)+x_{1, t_{0}}+e^{-\kappa\left(T_{1}-t_{0}\right)} x_{2, t_{0}}
\end{aligned}
$$

Using the coefficients estimated with the data up to December 31, 2007, I compute the price forecast, according to (8), for the futures contracts no more than 12 months to delivery (18 months to delivery for corn) that are traded over the period between January 1, 2008 and October 2, 2009. The forecast accuracy is compared between the two estimation methods by the root mean squared error (RMSE) of the resulting price forecasts.

Table 6 summarizes the results of forecasting test. In the table, the RMSE of the price forecast for crude oil is roughly identical for the two estimation methods. For the other three commodities, the forecast constructed with the model estimated by the sequential method attains substantially lower RMSE (by 13, 14, and 76 percent for natural gas, corn, and gold, 
respectively) than the forecast constructed with the model estimated by the simultaneous method.

\section{[TABLE 6 SOMEWHERE HERE]}

\section{Conclusion}

In this paper, I consider an alternative approach to the estimation of a term-structure model of commodity prices. In this approach, the model parameters are estimated in two steps; in the first step, the subset of model parameters is estimated on the first difference of futures prices; and, in the second step, the remaining model parameters, those determining seasonal mean price and deterministic variation in the common state variables, are estimated with a panel of futures prices while holding the other model parameters at their estimates from the first step. In the first step of this sequential procedure, first differencing eliminates deterministic price variation and the dynamics of the common state variables from the estimation equation and thus allows estimation of key model parameters without being affected by the bias in specifying these dynamics. The bias in specifying these dynamics, hence, can be quantified by comparing the model parameters estimated on the first difference form with the model estimated by the conventional approach of estimating all parameters simultaneously with a panel of futures prices.

Empirical estimations of a two-factor term-structure model widely considered in the literature with daily futures price data from four commodity markets reveal that the bias in specifying deterministic price variation and the dynamics of the state variables is of substantial magnitude. The variance of the measurement error estimated with the futures price equations is 17.6 to 102.4 times greater than the estimates obtained on the first difference form of the model. For gold, the simultaneous method yields a substantially higher estimate of the diffusion rate of the ST factor than the estimate obtained by the sequential method. These results indicate that the model estimated by the conventional method implies high price volatility of the four commodities, yet a dominant share of this high volatility represents the errors in specifying the stochastic dynamics of the underlying spot price. The magnitude of the upward bias in the estimated variance parameters questions the model's practicality in pricing various derivative contracts, especially options and other contracts whose pricing depends heavily on the volatility of the underlying asset value. 
The estimates of other model parameters also differ substantially between the two estimation methods. The estimated mean-reversion coefficient is slightly lower (implying higher persistence) for the simultaneous estimation than for the sequential estimation. The market price of risk parameters estimated by the simultaneous method are significant for some commodities while those estimated by the sequential method are insignificant for all four commodities. The sequential estimation yields substantially higher estimates of seasonal mean prices for natural gas and gold and slightly lower mean prices estimates for corn than the simultaneous estimation. The predicted values of the latent state variables differ substantially between the two estimation methods, in accordance with the difference in the estimated seasonal mean prices. Without observing the true stochastic dynamics, one cannot quantify the relative magnitudes of bias in the estimated model parameters by the two estimation methods. However, an advantage of the suggested sequential estimation method over the simultaneous method is illustrated by the out-of-sample forecast test, which indicates that for three of the four commodities the model estimated by the sequential method yields a substantially more accurate price forecast than the model estimated by the simultaneous method. 


\section{Acknowledgement}

I gratefully acknowledge insightful comments from two anonymous reviewers, which helped improve the paper. I also would like to thank Felix Chan and Aaron Smith for insightful discussions and Ellen Young for carefully proof reading the manuscript. This research is conducted with financial support from the Australian Research Council Discovery Grant (DP1094499).

\section{References}

[1] R. Bhar, D. Lee, Time-varying market price of risk in the crude oil futures market, Journal of Futures Markets 31(8) (2011) 779-807.

[2] J. Casassus, P. Collin-Dufresne, Stochastic convenience yield implied from commodity futures and interest rate, Journal of Finance 60 (2005) 2283-2331.

[3] C-L. Chang, M. McAleer, R. Tansuchat, Crude oil hedging strategies using dynamic multivariate GARCH, Energy Economics 33 (2011) 912-923.

[4] G. Cortazar, L. Naranjo, An N-factor Gaussian model of oil futures prices, Journal of Futures Markets 26(3) (2006) 243-268.

[5] G. Cortazar, E.S. Schwartz, Implementing a stochastic model for oil futures prices, Energy Economics 25 (2003) 215-238.

[6] R. Gibson, E.S. Schwartz, Stochastic convenience yield and the pricing of oil contingent claims, Journal of Finance 45(3) (1990) 959-976.

[7] J.C. Hull, Options, Futures, and Other Derivatives, $5^{\text {th }}$ ed., Prentice Hall, 2002.

[8] D. Lautier, Term structure models of commodity prices: A review, Journal of Alternative Investments 8(1) (2005) 42-64.

[9] Y-H. Lee, H-N. Hu, J-S. Chiou, Jump dynamics with structural breaks for crude oil prices, Energy Economics 32 (2010) 343-350.

[10] P. Liu, K. Tang, The stochastic behavior of commodity prices with heteroskedasticity in the convenience yield, Journal of Empirical Finance 18 (2011) 211-224.

[11] J. Lucia, E.S. Schwartz, Electricity prices and power derivatives: Evidence from the Nordic power exchange, Review of Derivatives Research 5 (2004) 5-50.

[12] M. Manoliu, S. Tompaidis, Energy futures prices: Term structure models with Kalman filter estimation, Applied Mathematical Finance 9 (2002) 21-43.

[13] K.R. Mitersen , E.S. Schwartz, Pricing of options on commodity futures with stochastic term structures of convenience yields and interest rates, Journal of Financial and Quantitative Analysis 33(1) (1998) 33-59.

[14] M.J. Nielsen, E.S. Schwartz, Theory of storage and the pricing of commodity claims, Review of Derivatives Research 7 (2004) 5-24. 
[15] B.R. Routledge, D.J. Seppi, C.S. Spatt, Equilibrium forward curves for commodities, Journal of Finance 55 (2000) 1297-1338.

[16] E.S. Schwartz, The Stochastic behavior of commodity prices: Implications for valuation and hedging, Journal of Finance 52 (1997) 923-973.

[17] E.S. Schwartz, J.E. Smith, Short-term variations and long-term dynamics in commodity prices, Management Science 46 (2000) 893-911.

[18] A. Smith, Partially overlapping time series: A new model for volatility dynamics for commodity futures, Journal of Applied Econometrics 20 (2005) 405-422.

[19] C. Sorensen, Modeling seasonality in agricultural commodity futures, Journal of Futures Markets 22(5) (2002) 393-426.

[20] H. Suenaga, Misspecification in term structure models of commodity prices: Implications for hedging price risk, in F. Chan, D. Marinova, R.S. Anderssen (eds.), MODSIM 2011, $19^{\text {th }}$ International Congress on Modelling and Simulation, Modelling and Simulation Society of Australia and New Zealand, December 2011, pp. 1652-1658.

[21] H. Suenaga, A. Smith, Volatility Dynamics and Seasonality in Energy Prices: Implications for Crack-Spread Price Risk, Energy Journal 32(3) (2011) 27-58.

[22] H. Suenaga, A. Smith, J.C. Williams, Volatility dynamics of NYMEX natural gas futures prices, Journal of Futures Markets 28 (2008) 438-463.

[23] K. Tang, Time-varying long-run mean of commodity prices and the modeling of futures term structures, Quantitative Finance, 12(5) (2012) 781-790.

[24] M.I. Todorova, Modeling energy commodity futures: Is seasonality part of it?, Journal of Alternative Investments Fall (2004) 10-31.

[25] J.C. Williams, B.D. Wright, Storage and Commodity Markets, Cambridge University Press, New York, 1991. 
Figure 1

Figure 1. Variance of idiosyncratic error as estimated by two methods for crude oil (a) Sequential estimation (Estimation on first difference form) (b) Simultaneous estimation (Estimation on futures price

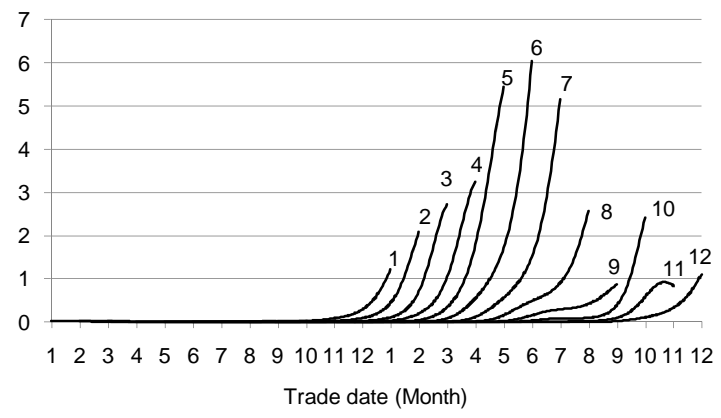

123345567889101112122345567889101112 Trade date (Month)

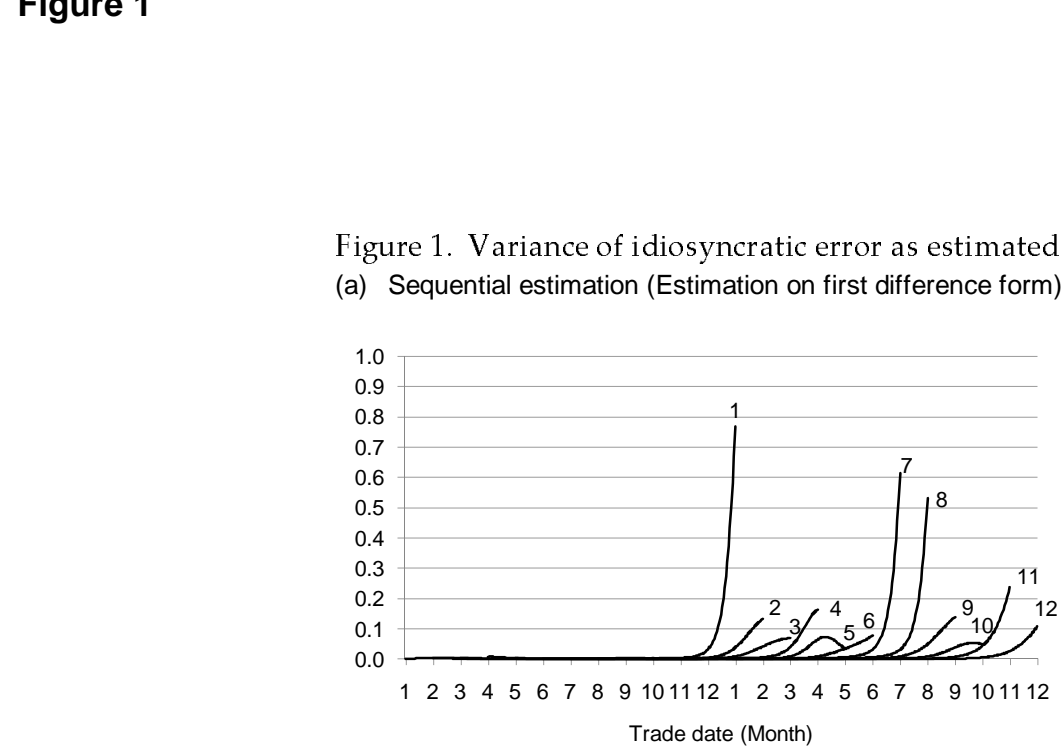


Figure 2

Figure 2. Variance of idiosyncratic error as estimated by two methods for natural gas

(a) Sequential estimation (Estimation on first difference form) (b) Simultaneous estimation (Estimation on futures price equations)
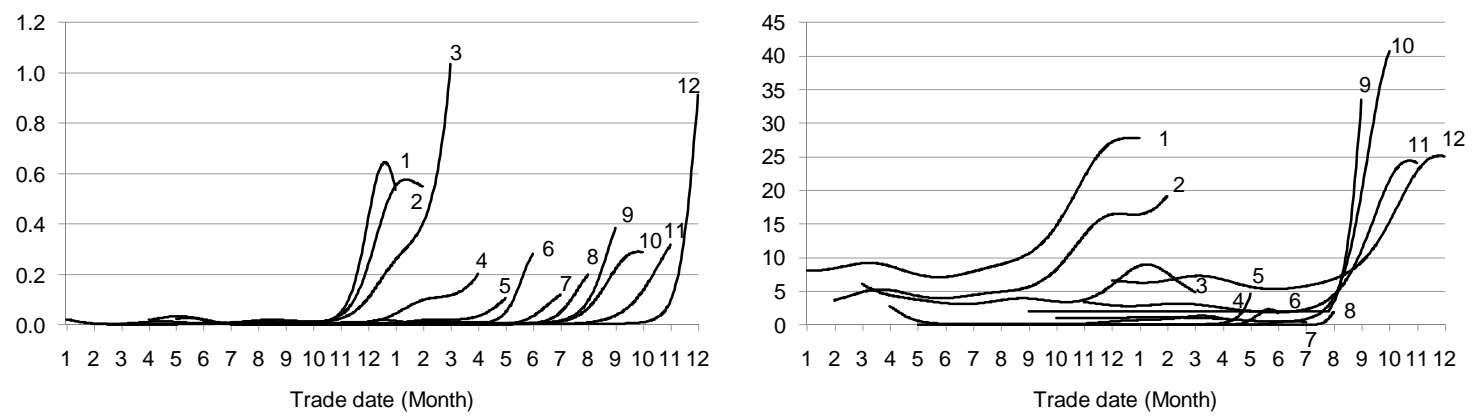
Figure 3

Figure 3. Variance of idiosyncratic error as estimated by two methods for gold (a) Sequential estimation (Estimation on first difference form)
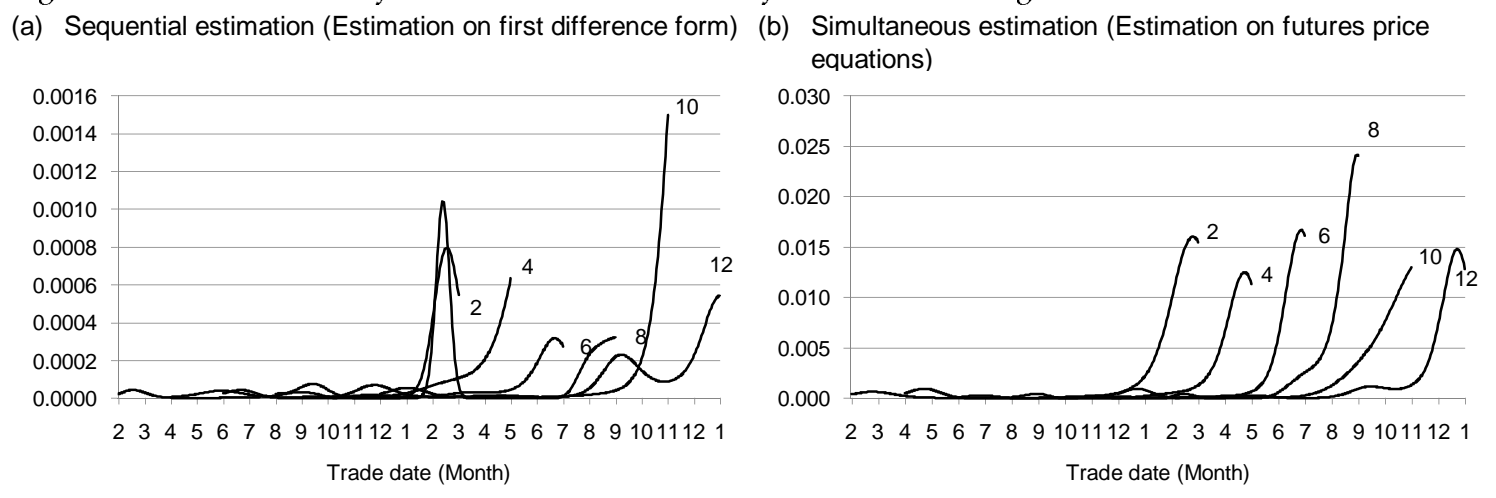
Figure 4. Variance of idiosyncratic error as estimated by two methods for corn

(a) Sequential estimation (Estimation on first difference form) (b) Simultaneous estimation (Estimation on futures price

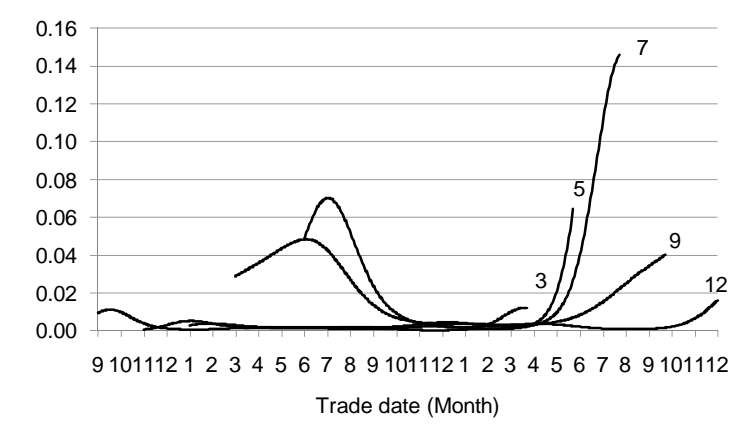

9101112123456789101112123456789101112 Trade date (Month)

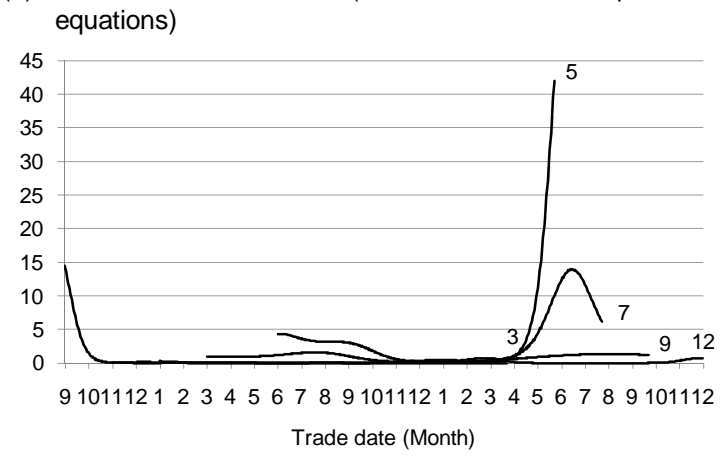

Figure 4 
Figure 5. Variance attributable to two common factors

(a) Crude Oil

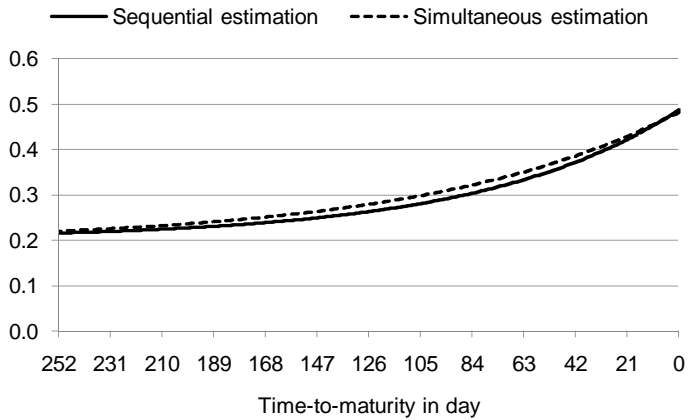

(b) Natural Gas

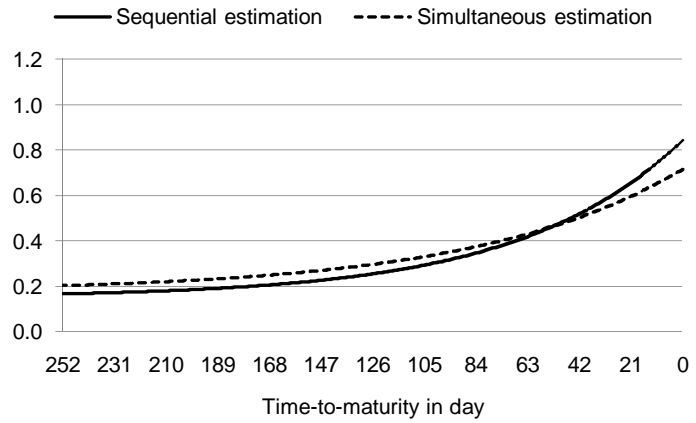

(c) Gold

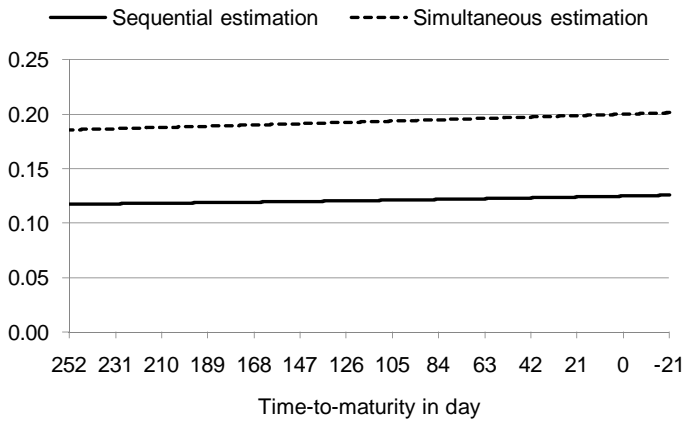

(d) Corn

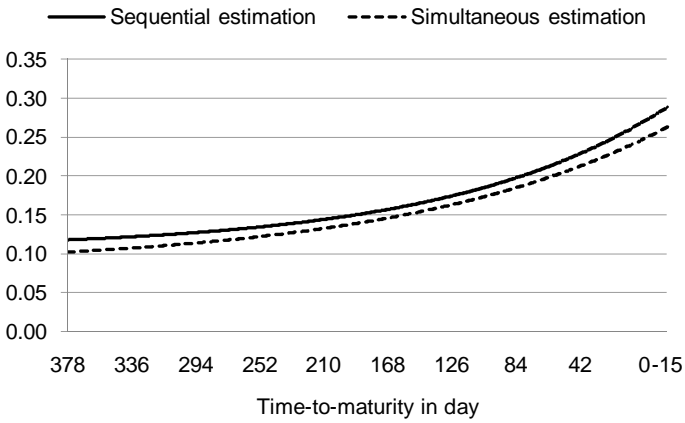


Table 1. Results of estimating two-factor Gaussian model by two estimation methods: Crude oil $^{+}$

\begin{tabular}{|c|c|c|c|c|c|c|}
\hline & \multicolumn{3}{|c|}{ Simultaneous estimation } & \multicolumn{3}{|c|}{ Sequential estimation } \\
\hline & Coefficient & Srd. Err. & $t$-ratio & Coefficient & Srd. Err. & $t$-ratio \\
\hline \multicolumn{7}{|c|}{ Seasonal mean price } \\
\hline January & 3.3937 & 0.0017 & 1989.319 & 3.4094 & 0.0053 & 646.754 \\
\hline February & 3.3936 & 0.0017 & 1996.421 & 3.4093 & 0.0053 & 646.685 \\
\hline March & 3.3931 & 0.0017 & 1999.079 & 3.4087 & 0.0053 & 646.606 \\
\hline April & 3.3928 & 0.0017 & 2003.313 & 3.4084 & 0.0053 & 646.434 \\
\hline May & 3.3923 & 0.0017 & 1999.074 & 3.4080 & 0.0053 & 646.476 \\
\hline June & 3.3918 & 0.0017 & 1999.023 & 3.4074 & 0.0053 & 646.314 \\
\hline July & 3.3913 & 0.0017 & 1989.323 & 3.4067 & 0.0053 & 645.834 \\
\hline August & 3.3910 & 0.0017 & 1982.357 & 3.4064 & 0.0053 & 645.707 \\
\hline September & 3.3914 & 0.0017 & 1984.037 & 3.4067 & 0.0053 & 645.661 \\
\hline October & 3.3919 & 0.0017 & 1985.926 & 3.4073 & 0.0053 & 645.970 \\
\hline November & 3.3930 & 0.0017 & 1990.900 & 3.4087 & 0.0053 & 646.509 \\
\hline December & 3.3938 & 0.0017 & 1990.796 & 3.4095 & 0.0053 & 646.662 \\
\hline \multicolumn{7}{|c|}{ Physical dynamics of common factors } \\
\hline$\mu \times 10^{4}$ & 0.1827 & 0.0365 & 5.004 & 0.1780 & 0.0040 & 44.896 \\
\hline$\sigma_{1}$ & 0.0140 & 0.0003 & 53.492 & 0.0142 & 0.0003 & 54.206 \\
\hline$\sigma_{2}$ & 0.0169 & 0.0007 & 25.340 & 0.0167 & 0.0007 & 23.622 \\
\hline$\phi\left(=e^{-\kappa}\right)$ & 0.9951 & 0.0001 & $1.73 E+04$ & 0.9938 & 0.0003 & $3.35 \mathrm{E}+03$ \\
\hline$\rho$ & 0.0000 & 0.0000 & 0.217 & 0.0132 & 0.0498 & 0.264 \\
\hline \multicolumn{7}{|c|}{ Market price of risks } \\
\hline$\lambda_{1} \times 10^{4}$ & 0.2862 & 0.0361 & 7.928 & 0.3367 & 0.9447 & 0.356 \\
\hline$\lambda_{2} \times 10^{4}$ & 0.3571 & 0.2572 & 1.389 & 0.3857 & 0.7082 & 0.545 \\
\hline
\end{tabular}

+ Estimates of the coefficients determining the variance of the measurement error are not included in this table.

* Parameters are estimated on the first difference model in the first stage of two-step estimation. 
Table 2. Results of estimating two-factor Gaussian model by two estimation methods: Natural gas ${ }^{+}$

\begin{tabular}{|c|c|c|c|c|c|c|}
\hline & \multicolumn{3}{|c|}{ Simultaneous estimation } & \multicolumn{3}{|c|}{ Sequential estimation } \\
\hline & Coefficient & Srd. Err. & $t$-ratio & Coefficient & Srd. Err. & $t$-ratio \\
\hline \multicolumn{7}{|c|}{ Seasonal mean price } \\
\hline January & 0.6568 & 0.2048 & 3.207 & 0.7947 & 0.0024 & 335.964 \\
\hline February & 0.6087 & 0.2049 & 2.970 & 0.7481 & 0.0022 & 336.686 \\
\hline March & 0.5459 & 0.2061 & 2.648 & 0.6875 & 0.0021 & 321.506 \\
\hline April & 0.4753 & 0.2048 & 2.321 & 0.6143 & 0.0018 & 341.968 \\
\hline May & 0.4635 & 0.2046 & 2.265 & 0.6026 & 0.0018 & 342.845 \\
\hline June & 0.4657 & 0.2047 & 2.275 & 0.6048 & 0.0018 & 340.660 \\
\hline July & 0.4705 & 0.2047 & 2.299 & 0.6097 & 0.0018 & 330.303 \\
\hline August & 0.4773 & 0.2047 & 2.331 & 0.6169 & 0.0019 & 326.274 \\
\hline September & 0.4829 & 0.2048 & 2.358 & 0.6230 & 0.0019 & 325.493 \\
\hline October & 0.5023 & 0.2047 & 2.454 & 0.6434 & 0.0019 & 340.332 \\
\hline November & 0.5665 & 0.2050 & 2.764 & 0.7068 & 0.0020 & 352.435 \\
\hline December & 0.6321 & 0.2048 & 3.086 & 0.7712 & 0.0023 & 340.974 \\
\hline \multicolumn{7}{|c|}{ Physical dynamics of common factors } \\
\hline$\mu \times 10^{4}$ & 0.3635 & 0.1235 & 2.943 & 0.3322 & 0.0212 & 15.684 \\
\hline$\sigma_{1}$ & 0.0132 & 0.0002 & 53.018 & 0.0123 & 0.0002 & $54.331 *$ \\
\hline$\sigma_{2}$ & 0.0232 & 0.0005 & 50.515 & 0.0263 & 0.0005 & $54.932 *$ \\
\hline$\phi\left(=e^{-\kappa}\right)$ & 0.9941 & 0.0002 & 5432.031 & 0.9925 & 0.0004 & $2.31 \mathrm{E}+03 *$ \\
\hline$\rho$ & 0.0000 & 0.0004 & 0.063 & 0.0000 & 0.0002 & $0.149 *$ \\
\hline \multicolumn{7}{|c|}{ Market price of risks } \\
\hline$\lambda_{1} \times 10^{4}$ & 0.6747 & 0.1267 & 5.324 & 0.5556 & 0.3187 & $1.743 *$ \\
\hline$\lambda_{2} \times 10^{4}$ & -0.2838 & 0.1861 & -1.525 & -0.3295 & 0.3429 & -0.961 \\
\hline
\end{tabular}

+ Estimates of the coefficients determining the variance of the measurement error are not included in this table.

* Parameters are estimated on the first difference model in the first stage of two-step estimation.

Table 3. Results of estimating two-factor Gaussian model by two estimation methods: Gold ${ }^{+}$

\begin{tabular}{|c|c|c|c|c|c|c|}
\hline & \multicolumn{3}{|c|}{ Simultaneous estimation } & \multicolumn{3}{|c|}{ Sequential estimation } \\
\hline & Coefficient & Srd. Err. $\times 10^{2}$ & $t$-ratio & Coefficient & Srd. Err. & $t$-ratio \\
\hline \multicolumn{7}{|c|}{ Seasonal mean price } \\
\hline February & 6.3824 & 0.0042 & $1.53 \mathrm{E}+05$ & 6.7263 & 0.1082 & 62.186 \\
\hline April & 6.3824 & 0.0040 & $1.58 \mathrm{E}+05$ & 6.7265 & 0.1082 & 62.187 \\
\hline June & 6.3825 & 0.0039 & $1.66 \mathrm{E}+05$ & 6.7265 & 0.1082 & 62.188 \\
\hline August & 6.3825 & 0.0039 & $1.63 \mathrm{E}+05$ & 6.7264 & 0.1082 & 62.187 \\
\hline October & 6.3823 & 0.0039 & $1.62 \mathrm{E}+05$ & 6.7263 & 0.1082 & 62.186 \\
\hline December & 6.3823 & 0.0041 & $1.56 \mathrm{E}+05$ & 6.7263 & 0.1082 & 62.186 \\
\hline \multicolumn{7}{|c|}{ Physical dynamics of common factors } \\
\hline$\mu \times 10^{4}$ & -0.1063 & 3.0621 & -3.471 & -0.0042 & 0.0026 & -1.584 \\
\hline$\sigma_{1}$ & 0.0110 & 0.0220 & 50.287 & 0.0105 & 0.0002 & $59.251 *$ \\
\hline$\sigma_{2}$ & 0.0088 & 0.0197 & 44.968 & 0.0039 & 0.0002 & $20.467 *$ \\
\hline$\phi\left(=e^{-\kappa}\right)$ & 0.9996 & 0.0008 & $1.26 \mathrm{E}+05$ & 0.9986 & 0.0001 & $1.09 \mathrm{E}+04 *$ \\
\hline$\rho$ & 0.0000 & 0.0193 & 0.131 & 0.0000 & 0.0006 & $0.041 *$ \\
\hline \multicolumn{7}{|c|}{ Market price of risks } \\
\hline$\lambda_{1} \times 10^{4}$ & -0.3744 & 3.0777 & -12.164 & -0.1991 & 0.3347 & $-0.595 *$ \\
\hline$\lambda_{2} \times 10^{4}$ & -0.2036 & 0.8914 & -22.840 & 0.0975 & 0.2111 & $0.462 *$ \\
\hline
\end{tabular}

+ Estimates of the coefficients determining the variance of the measurement error are not included in this table.

* Parameters are estimated on the first difference model in the first stage of two-step estimation. 
Table 4. Results of estimating two-factor Gaussian model by two estimation methods: Corn ${ }^{+}$

\begin{tabular}{|c|c|c|c|c|c|c|}
\hline & \multicolumn{3}{|c|}{ Simultaneous estimation } & \multicolumn{3}{|c|}{ Sequential estimation } \\
\hline & Coefficient & Srd. Err. $\times 10^{2}$ & $t$-ratio & Coefficient & Srd. Err. & $t$-ratio \\
\hline \multicolumn{7}{|c|}{ Seasonal mean price } \\
\hline March & 5.9352 & 0.5515 & 1076.205 & 5.8221 & 0.0585 & 99.587 \\
\hline May & 5.9458 & 0.5519 & 1077.285 & 5.8323 & 0.0584 & 99.814 \\
\hline July & 5.9503 & 0.5527 & 1076.501 & 5.8367 & 0.0585 & 99.727 \\
\hline September & 5.9271 & 0.5500 & 1077.609 & 5.8141 & 0.0581 & 100.013 \\
\hline November & 5.9169 & 0.5563 & 1063.539 & 5.8039 & 0.0583 & 99.521 \\
\hline \multicolumn{7}{|c|}{ Physical dynamics of common factors } \\
\hline$\mu \times 10^{4}$ & 0.0456 & 0.8687 & 5.243 & -0.0018 & 0.0324 & -0.056 \\
\hline$\sigma_{1}$ & 0.0092 & 0.0154 & 59.420 & 0.0103 & 0.0002 & 68.347 \\
\hline$\sigma_{2}$ & 0.0128 & 0.0222 & 57.643 & 0.0128 & 0.0003 & 40.859 \\
\hline$\phi\left(=e^{-\kappa}\right)$ & 0.9971 & 0.0090 & $1.11 \mathrm{E}+04$ & 0.9965 & 0.0001 & $1.24 \mathrm{E}+04$ \\
\hline$\rho$ & 0.0000 & 0.0025 & 0.428 & 0.0000 & 0.0001 & 0.183 \\
\hline \multicolumn{7}{|c|}{ Market price of risks } \\
\hline$\lambda_{1} \times 10^{4}$ & 0.1742 & 1.8421 & 9.459 & 0.0884 & 0.0761 & 1.163 \\
\hline$\lambda_{2} \times 10^{4}$ & -0.0794 & 5.8130 & -1.366 & -0.3788 & 0.2848 & -1.330 \\
\hline
\end{tabular}

+ Estimates of the coefficients determining the variance of the measurement error are not included in this table.

* Parameters are estimated on the first difference model in the first stage of two-step estimation.

Table 5. Sample statistics of the predicted latent factors

\begin{tabular}{|c|c|c|c|c|c|}
\hline & & \multicolumn{2}{|c|}{ Simultaneous estimation } & \multicolumn{2}{|c|}{ Sequential estimation } \\
\hline & & Mean & Std. dev. & Mean & Std. dev. \\
\hline \multicolumn{6}{|l|}{ Crude oil } \\
\hline & $x_{1}$ & -0.177 & 0.444 & -0.175 & 0.444 \\
\hline & $x_{2}$ & 0.015 & 0.154 & -0.003 & 0.144 \\
\hline \multicolumn{6}{|l|}{ Natural gas } \\
\hline & $x_{1}$ & 0.634 & 0.605 & 0.471 & 0.595 \\
\hline & $x_{2}$ & -0.025 & 0.156 & -0.002 & 0.247 \\
\hline \multicolumn{6}{|l|}{ Gold } \\
\hline & $x_{1}$ & -1.331 & 0.442 & -0.755 & 0.265 \\
\hline & $x_{2}$ & 0.879 & 0.342 & -0.042 & 0.114 \\
\hline \multicolumn{6}{|l|}{ Corn } \\
\hline & $x_{1}$ & -0.287 & 0.151 & -0.294 & 0.152 \\
\hline & $x_{2}$ & -0.138 & 0.158 & -0.019 & 0.172 \\
\hline
\end{tabular}

Table 6. Results of out-of-sample forecast test: RMSE of the forecast for the period between Jan 1, 2008 and Oct 2, 2009

\begin{tabular}{lrrr}
\hline & $\begin{array}{r}\text { Simultanous } \\
\text { estimation }\end{array}$ & $\begin{array}{r}\text { Sequential } \\
\text { estimation }\end{array}$ & $\begin{array}{r}\text { Sequential in \% } \\
\text { of Simultaneous }\end{array}$ \\
\hline Crude oil & 0.155 & 0.159 & $103 \%$ \\
Natural gas & 0.232 & 0.201 & $87 \%$ \\
Gold & 0.039 & 0.009 & $24 \%$ \\
Corn & 0.058 & 0.049 & $86 \%$ \\
\hline
\end{tabular}

